\title{
Evolution of Quantitative Characteristics Which are Determined by Several Additive Loci
}

\author{
FRANK B. LIVINGSTONE \\ Department of Anthropology, University of Michigan, \\ Ann Arbor, Michigan 48104
}

\begin{abstract}
For several models of polygenic traits due to equal and additive alleles the rate of change in allele frequency was estimated. With an amount of selection comparable to the present in several modern studies, evolution appears to be quite fast; so that the rapid change from Neanderthal to modern man could have been accomplished in a very short time evolutionarily speaking.
\end{abstract}

Most of the biological characteristics which have been considered in this symposium are polygenic or due to the action of several loci. The variation in these traits can also be due to environmental factors, although the amount of environmental influence varies considerably. For linear measurements on bone or on living subjects the environmental influence and hence the heritability averages about $50 \%$. In recent years several approaches to the analysis of polygenic traits have been developed, principally by geneticists working with organisms other than man; and many are the result of the analytic capabilities of the computer. This paper attempts to apply some of these methods to the analysis of human evolution.

In any breeding population the variation among the adults of a single sex for most linear measurements is normally distributed. If the genetic component of the trajt is due to several loci, and their effects are equal and additive, then the genetic variation in the trait would approach a normal distribution as the number of loci is increased. In this paper the loci are assumed to be equal and additive and to have two different types of alleles, plus and minus, or $\mathrm{A}$ and $\mathrm{a}$, one of which (A) increases the measurement and one (a) which does not. Thus, the three genotypes, aa, $\mathrm{Aa}$, and $\mathrm{AA}$ contribute zero, one and two units to the trait, respectively. If the environmental influences are also normally distributed and independent of the genetic effects, then the phenotype variation would be a combination of two independent normal distributions and hence would also be expected to be nor mally distributed as in fact it is.

This normal distribution will actually be expected at birth with random mating, equal fertility, and no gametic disequilibrium. The action of selection through differential mortality of the resulting phenotypes will tend to change the distribution. Of course, selection can also act through differential fertility. Most selection tends to weed out or act against the extremes, so that the frequencies of the most divergent classes will be less than expected. However, with the usual amount of selection in natural populations, this change will be minimal. For most metrical. characteristics which have remained relatively constant in human populations for a number of generations, the most generally assumed model has the optimum fitness close to the mean. If fitness decreases equally with distance from the mean, both larger and smaller, then selection will stabilize the distribution, although with certain assumptions there can still be some gene frequency change. On the other hand if fitness decreases unequally on either side of the mean or the optimum fitness is different from the mean, selection will tend to change the mean and the distribution of the trait. Most studies assume fitness to vary as the square of the distance from the mean, but here fitness will be assumed for the most part to vary linearly with the distance from the mean.

For human populations there are few studies which have attempted to measure variation in fitness for metric traits. It 
would seem unlikely, however, that the differences in fitness have a maximum of more than $10 \%$. Phenotypes due to major mutations such as achondroplasia have a much lower fitness, which has been estimated to be about $10 \%$ of that of normals (Saldanha, '62). Karn and Penrose's classic study of birthweight ('51) found that infants with birthweights between 7.5 and 8.5 pounds survive $98.5 \%$ of the time, while those weighing more than 10 pounds survive $90.5 \%$. This implies a fitness of about $92 \%$ that of average birthweight, and the same decrease in fitness is found for infants with birthweights lighter than the mean. Based on the number of siblings and their survival, the fitnesses associated with cephalic index have been estimated by Bielicki and Welon ('64) in Poland and by Huizenga and Slob ('65) in the Netherlands. In both cases the mesocephalics had the highest fitness, but in Poland the ultra-dolichocephalics had the lowest fitness of 0.93 and the ultra-brachycephalics had 0.982, while in the Netherlands the brachycephalics had the lowest fitness of 0.965 . Studies in the United States on Yale men (Huntingdon, '45) and on Harvard men (Damon and Thomas, ' 67 ) both indicate that short men have fewer children. Damon and Thomas found that Harvard men under $5^{\prime} 7^{\prime \prime}$ tall had $96 \%$ as many children as those between $5^{\prime} 7^{\prime \prime}$ and $5^{\prime} 9^{\prime \prime}$, while those over $6^{\prime}$ tall had $97 \%$ as many children. In most cases these fitness differences are not significant, but they do point in the same direction and will be used as a general approximation to the amount of selection which can occur for a metric trait.

Selection also has more effect on the variance of a metric trait than on the mean. Haldane ('59) showed that selection against hemophilics changes the mean clotting time by only $0.027 \%$ but it changes the variance by $2.3 \%$. In the Parma River Valley of Italy Cavalli-Sforza ('62) found the variance of stature among unmarried adults to be significantly greater than that of married adults.

To illustrate the process of evolution as it has occurred in the human species, the distributions of two cranial measurements for the Neanderthal finds and an early sapiens group are shown on figure 1. The Neanderthal measurements were taken from Coon ('62), and the sapiens, from a Mesolithic people of Northwest Africa given in Briggs ('55: 39-40). These two measurements were selected because they seem to differ the most between the two groups and have been recorded for an appreciable number of Neanderthal crania. There are 9 and 12 individuals, respectively in the Neanderthal series for basion-bregma and cranial length, while there are 15 and 29, respectively, for the sapiens group. Obviously the Neanderthal finds did not constitute a single population, but neither did the sapiens group although they were surely much closer in time and space. It can be seen that the two groups only differ by 2 or 3 standard deviations for both measurements. The standard deviations of the two groups are somewhat different but not significantly so, and these standard deviations do not appear to be very different from those of actual populations. However, even with these small samples the means of the two populations are very significantly different; so that evolution has occurred. The questions are thus how much and how long would it take.

Assuming these measurements are due to either 4 or 8 loci, an approximation to the two distributions with 4 loci is shown on figure 2 and with 8 loci on figure 3 . If the frequencies of the minus alleles for all loci are changed from 0.75 to 0.25 , the genotype distribution will change as indicated by the arrows and about as much as the difference in the distributions of the Neanderthal and sapiens group for the two cranial measurements. In fact even more change occurs with 8 loci. Some of this change between Neanderthal and sapiens could be due to environmental effects, comparable to those which have increased the average stature of many human populations in the last century. For example, the stature of Swedish males has increased by five inches, which is about two standard deviations (Chamla, '64). Very little gene change could have occurred in this time. However, we will assume that the changes are due to gene frequency differences. 


\section{CRANIAL DIFFERENCES}
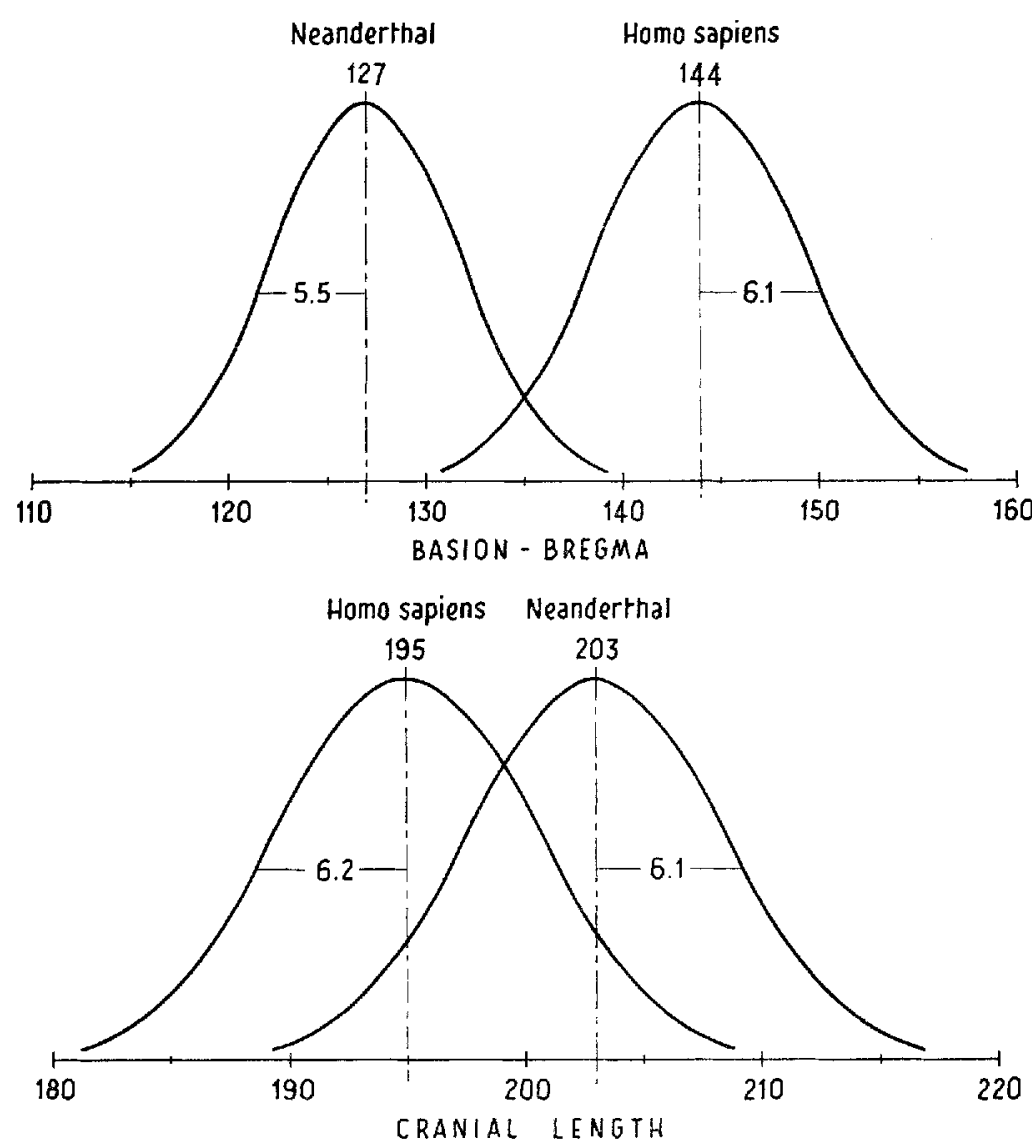

Fig. 1 The distribution of two cranial measurements for a group of Neanderthal finds and a group of prehistoric sapiens finds from Northwest Africa. Note: the distributions have not been tested for normality but only assumed to be.

The fitnesses of the various genotypes have been estimated by two sets of values for four loci and by one set for eight loci. These are shown on figures 2 and 3 . Fitness has been assumed to vary linearly from one optimum genotype, which has been assigned a fitness of 1.0. Most optimum models of selection have assumed fitness to vary with the square of the distance from the optimum (Lewontin, '64), and more recently Van Valen and Mellin ('67) have found that for birthweight fitness varies exponentially. Some runs were made with fitness varying as the square of the distance from the optimum, and the rate of evolution was in general comparable to the linear model. In addition, the computer program was run assuming random mating and gametic equilibrium. Some runs have been made for four loci which include the calculation of gametic disequilibrium, and, as Wright ('67) has shown, with no linkage and with the amount of selection usually found in natural conditions, gametic disequilibrium has minimal evolutionary consequences.

The results for the linear model are shown on figure 4 . When fitness varies by $12 \%$, it takes about 150 generations or perhaps 3500 years for the change to occur, while for a fitness variation of $6 \%$ it takes about twice as long or about 300 generations. With eight loci there are more genotypes, but with a comparable 


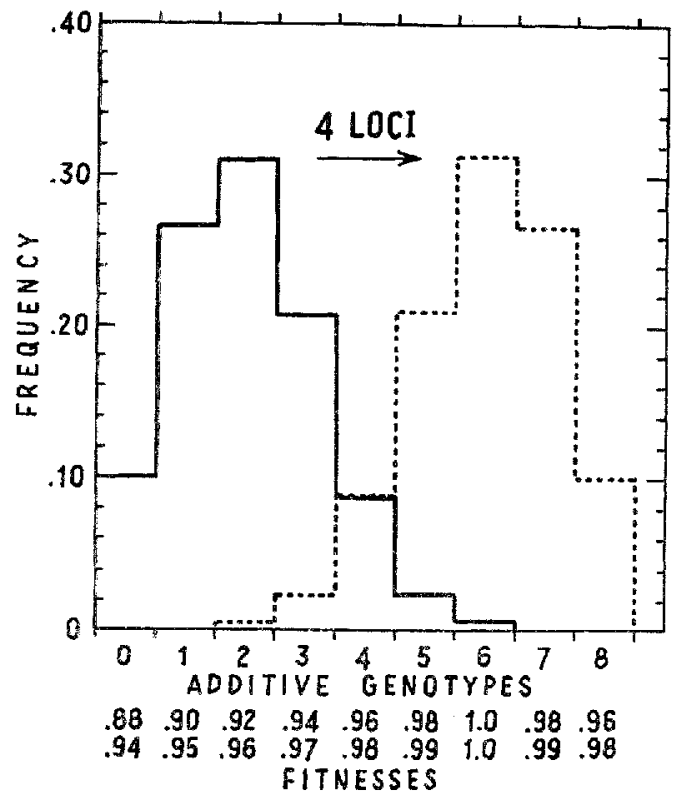

Fig. 2 The frequency distributions of the ad. ditive genotypes for four interacting loci with tandom mating and the minus alleles at a frequency of $0.25(----)$ and at $0.75(-)$. decrease of $1 \%$ in fitness with each unit of genotype variation away from the optimum, the rate of evolution is almost identical to that for four loci. The 8 loci run was not continued to equilibrium because of the expense involved and the fact that it did not seem to be any different.

All of the runs shown on figure 4 assume equal and additive loci and no dominance. Under these conditions, there is no stable equilibrium with both alleles present and with an optimum selection model (Wright, '35). The fact that the minus alleles were all started with the same frequencies is responsible for the changes being identical for all loci. But if the loci are begun with different allelic frequencies, some will fix for the minus allele and others for the plus allele. The number which fix either way will depend on the optimum genotype. Figure 5 shows this process when the optimum genotype is exactly intermediate between the extremes. For two different sets of fitness values two minus alleles were begun at one frequency and the two others at a

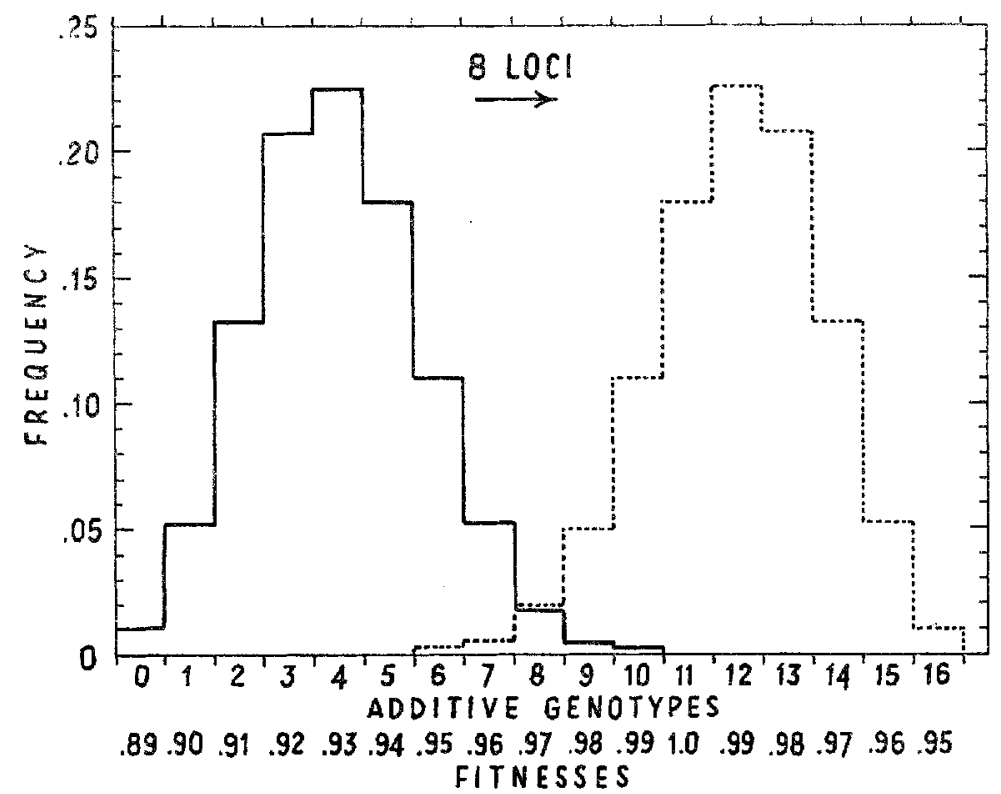

Fig. 3 The frequency distributions of the additive genotypes for eight interacting loci with random mating and the minus alleles at a frequency of $0.25(-\ldots)$ and at 0.75 $(\stackrel{-}{\longrightarrow}$. 


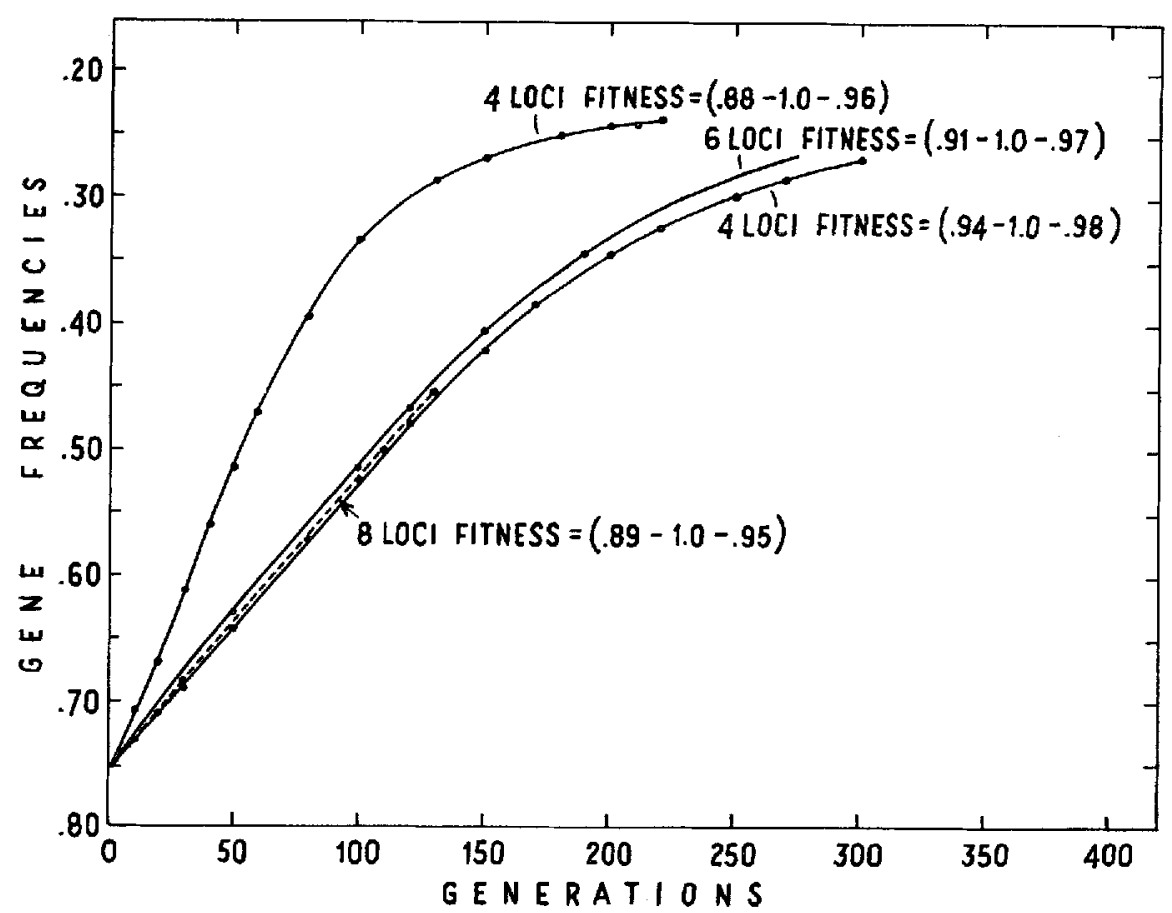

Fig. 4 The evolution of the minus allele frequencies for various numbers of interacting loci under the influence of selection. The fitness values for 4 and 8 loci are shown on figures 2 and 3 and the direction of change is shown by the arrow.

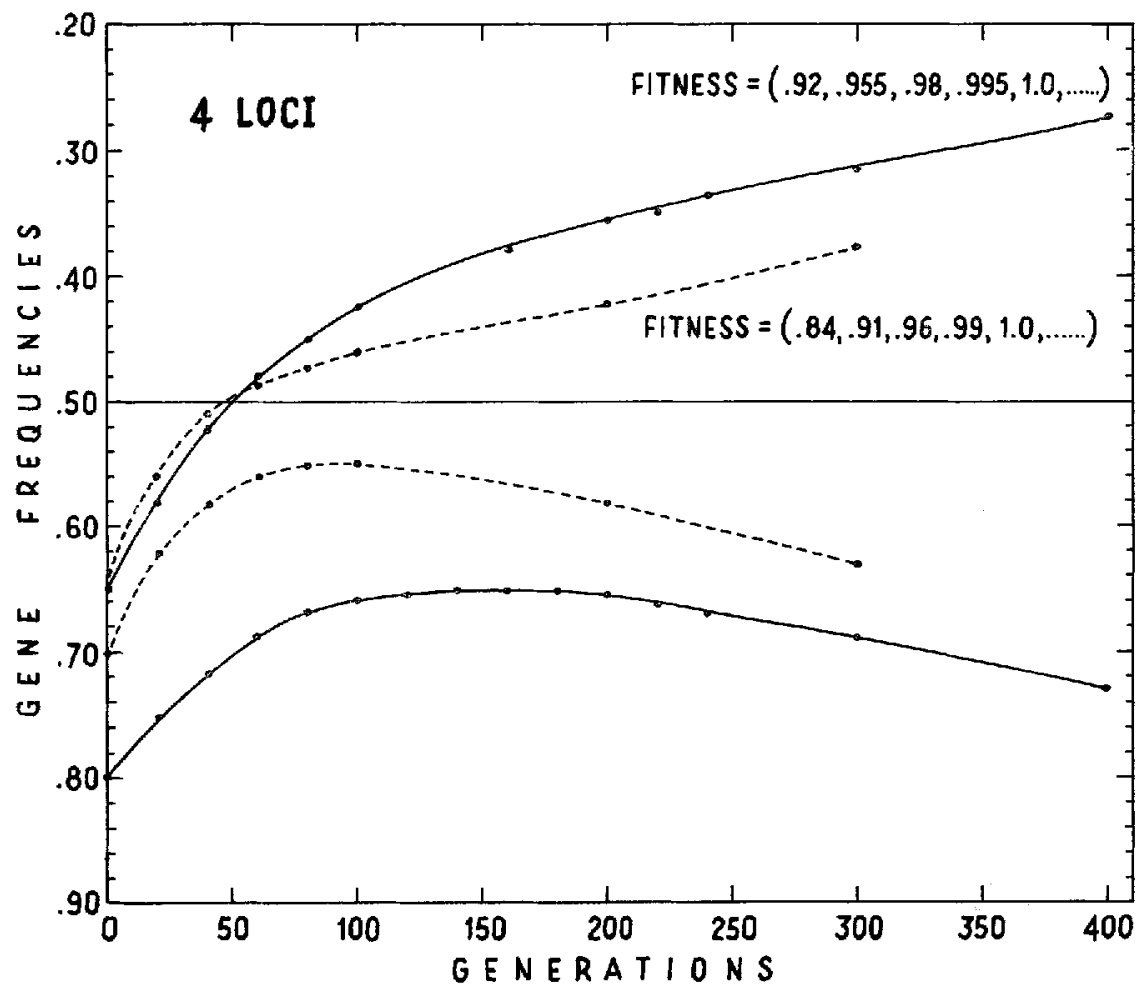

Fig. 5 The evolution of the minus allele frequencies toward fixation or elimination for 4 interacting loci and an optimum selection model. One run is represented by $(----)$ with two minus alleles at each line, while another run is represented by $(\longrightarrow)$ again with two minus alleles at each line. 
$4 \mathrm{LOCI}$

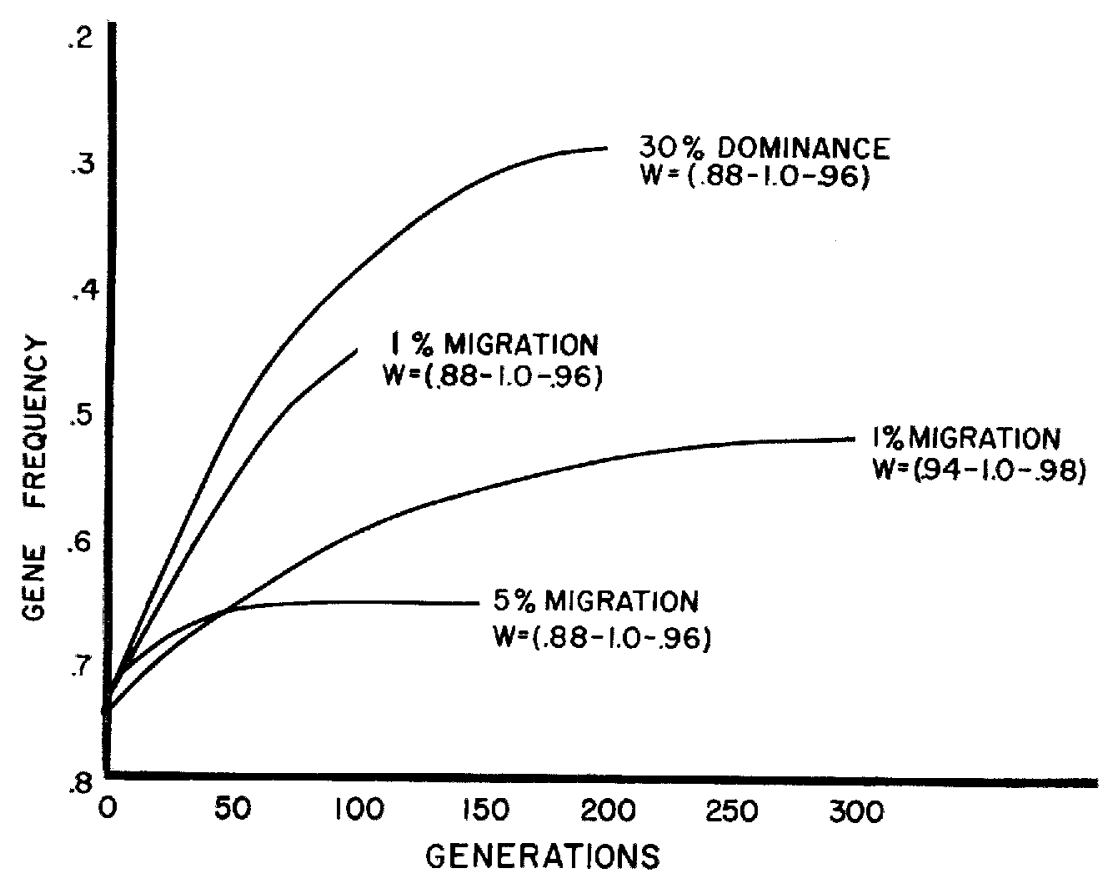

Fig. 6 The evolution of the minus allele frequencies with the same selection as in figure 4 but with the addition of dominance or migration.

different frequency. At first the frequencies rapidly change to a point when the average frequency of the minus alleles is 0.5 . Then at a slower rate the minus alleles which have frequencies greater than 0.5 begin to fix in the population, and those less than 0.5, to disappear. Of course, this is only true with this optimum genotype when these two types of minus alleles are equally frequent. If the optimum genotype is closer to the largest genotype, then fewer minus alleles will fix, while the opposite is true if it is closer to the smallest genotype. These results are all in accord with Robertson's ('56) analysis, and this replacement would have occurred in the runs illustrated on figure 4 if the allelic frequencies had not been equal for all loci. It should be noted, however, that on figure 5 fitness does not vary linearly but as the square of the distance from the optimum, but the variation in fitness is comparable to the linear models.

The effects of dominance, migration, and mutation were also examined, and some of the results are shown on figure 6. It can be seen that dominance of the order which Fisher ('18) found for human metric traits increases the rate of evolution when selection is increasing the dominant allele. Although no stable equilibria are present with no dominance or complete dominance, they can exist for intermediate dominance (Kojima, '59). Later runs were made with $80 \%$ dominance, which is within the range of stability for 4 loci according to Lewontin's ('64) extension of Kojima's results; and the frequencies appeared to be stable although there were still small changes in gene frequency after several hundred generations.

Migration was assumed to have originated from a population with the minus allele at every locus having a frequency of 0.75 . For a migration rate of $5 \%$ the effects of selection with the fitnesses varying by $12 \%$ were almost completely overwhelmed, and the minus allele frequencies stayed above 0.60 . But if migration was set at $1 \%$, then the minus allele 
frequencies changed at almost the same rate as they did with no migration. On the other hand, if fitness only varies by $6 \%$, then a migration rate of $1 \%$ has a considerable effect on the gene frequencies. Hence it would seem that more isolation than is usual among neighboring human isolates is necessary for genetic differences to develop with this amount of selection present. However, the great geographic dispersion of the human species could lead to the requisite isolation.

To estimate the effect of mutation, the fitnesses were all set equal to 1.0 and a mutation rate to minus alleles of $10^{-4}$ for each locus was introduced. When the minus alleles were begun at 0.25 , their frequencies were only increased by 0.005 in 100 generations. This implies a time span of 10,000 generations to evolve from 0.25 to 0.75 or vice-versa, which would be at least 200,000 years for human populations. Thus, the evolution of sapiens from Neanderthal man seems to be determined by other factors and the effects of mutation minimal if the short chronology is accepted. However, the estimate of the mutation rates is based on those known for single gene traits-for which they are generous--and loci determining metric traits may be more mutable. In any case, with the amount of selection apparently present in human populations, the evolution of Neanderthal to sapiens could have occurred in the time between perhaps 50,000 and 30,000 B.C.

\section{LITERATURE CITED}

Bielicki, T., and Z. Welon 1964 The operation of natural selection on human head form in an East European population. Homo, 15: 2230.

Briggs, L. C. 1955 The Stone Age Races of Northwest Africa. Bulletin 18, American School of Prehistoric Research, Peabody Museum.
Cavilli-Sforza, L. I. 1962 Demographic attacks on genetic problems: some possibilities and results. Seminar on the Use of Vital and Health Statistics for Genetic and Radiation Studies, Geneva 1960, United Nations, New York, pp. 221-230.

Chamla, M-C. 1964 L'accroissement de la stature en France de 1800 a 1960 ; comparaison avec les pays d'Europe Occidentale. Bull. Mem. Soc. Anthrop. Paris, Ser. 11, 6: 201-278.

Coon, C. S. 1962 The Origin of Races. Knopf, New York.

Damon, A., and R. B. Thomas 1967 Fertility and physique-height, weight, and ponderal index. Hum. Biol., 39: 5-13.

Fisher, R. A. 1918 The correlation between relatives on the supposition of Mendelian inheritance. Trans. Roy. Soc. Edinburgh, 52: 399-433.

Haldane, J. B. S. 1959 Natural selection. In: Darwin's Biological Work. P. R. Bell, ed. Cambridge University Press, Cambridge, pp. 101149.

Huizinga, J., and A. Slob 1965 Progressive brachycephalization: reproduction and headform in the Netherlands. Proc. Konink. Nederl. Akad. Wetensch., Ser. C, 68: 297-301.

Huntington, E. 1945 Mainsprings of Civilization. J. Wiley, New York.

Karn, M. N., and L. S. Penrose 1951 Birth weight and gestation time in relation to maternal age, parity and infant survival. Ann. Eugen., 15: 306-322.

Kojima, K-I. 1959 Stable equilibria for the optimum model. Proc. Nat. Acad. Sci., 45: 989-993.

Lewontin, R. C. 1964 The interaction of selection and linkage. II. Optimum models. Genetics, 50: 757-782.

Robertson, A. 1956 The effect of selection against extreme deviants based on deviation or on homozygosis. J. Genet., 54: 236-248.

Saldanha, P. H. 1962 The estimation of relative fitness of gene determining achondroplasia based on the equilibrium between mutation and selective elimination rate. J. Genet. Hum., 11: 314-324.

Van Valen, L., and G. W. Mellin 1967 Selection in natural populations. 7. New York babies (fetal life study). Ann. Hum. Genet., 31: 109127.

Wright, S. 1935 Evolution in populations in approximate equilibrium. J. Genet., 30: 257-266. 1967 Surfaces of selective value. Proc. Nat. Acad. Sci., 58: 165-172. 